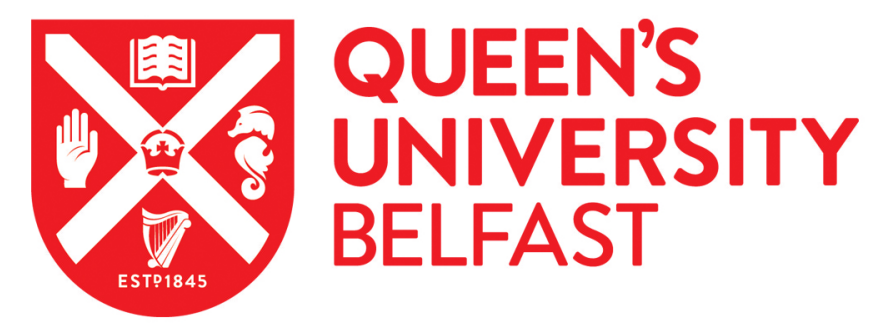

\title{
M-L band X-rays (3-3.5 KeV) from palladium coated targets for isochoric radiative heating of thin foil samples
}

Kettle, B., Dzelzainis, T., White, S., Li, L., Rigby, A., Spindloe, C., Notley, M., Heathcote, R., Lewis, C. L. S., \& Riley, D. (2015). M-L band x-rays (3-3.5 KeV) from palladium coated targets for isochoric radiative heating of thin foil samples. Journal of Physics B: Atomic Molecular and Optical Physics, 48(22), [224002].

https://doi.org/10.1088/0953-4075/48/22/224002

Published in:

Journal of Physics B: Atomic Molecular and Optical Physics

Document Version:

Peer reviewed version

Queen's University Belfast - Research Portal:

Link to publication record in Queen's University Belfast Research Portal

Publisher rights

(C) 2015 IOP Publishing Ltd

\section{General rights}

Copyright for the publications made accessible via the Queen's University Belfast Research Portal is retained by the author(s) and / or other copyright owners and it is a condition of accessing these publications that users recognise and abide by the legal requirements associated with these rights.

Take down policy

The Research Portal is Queen's institutional repository that provides access to Queen's research output. Every effort has been made to ensure that content in the Research Portal does not infringe any person's rights, or applicable UK laws. If you discover content in the Research Portal that you believe breaches copyright or violates any law, please contact openaccess@qub.ac.uk. 


\title{
M-L band x-rays (3-3.5keV) from palladium-coated targets for isochoric radiative heating of thin foil samples
}

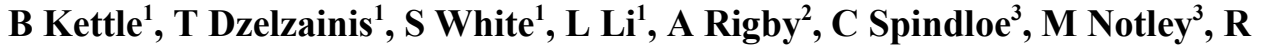 \\ Heathcote $^{3}$, C L S Lewis ${ }^{1}$, and D Riley ${ }^{1}$ \\ ${ }^{1}$ School of Mathematics and Physics, Queens University of Belfast, BT7 1NN, Belfast, UK \\ ${ }^{2}$ Clarendon Laboratory, Parks Road, Oxford, OX1 3PU, UK \\ ${ }^{3}$ Central Laser Facility, Rutherford Appleton Laboratory, Harwell, Oxfordshire, OX11 0QX, UK \\ E-mail: bkettle01@qub.ac.uk
}

\begin{abstract}
We describe experiments designed to produce a bright M-L band x-ray source in the 3-3.5keV region. Palladium targets irradiated with a $10^{15} \mathrm{~W} / \mathrm{cm}^{2}$ laser pulse have previously been shown to convert up to $\sim 2 \%$ of the laser energy into $\mathrm{M}-\mathrm{L}$ band $\mathrm{x}$-rays with similar pulse duration to that of the incident laser. This x-ray emission is further characterized here, including pulse duration and source size measurements, and a higher conversion efficiency than previously achieved is demonstrated $(\sim 4 \%)$ using more energetic and longer duration laser pulses (200ps). The emission near the aluminium K-edge (1.465-1.550keV) is also reported for similar conditions, along with the successful suppression of such lower band x-rays using a $\mathrm{CH}$ coating on the rear side of the target. The possibility of using the source to radiatively heat a thin aluminium foil sample to uniform warm dense matter conditions is discussed.
\end{abstract}

\section{Introduction}

The method of radiatively heating a target with a separate laser generated x-ray source is a widely used technique. Absorption of the laser energy and subsequent electron heat flow into the target creates a hot dense plasma that has a high ionization state and emits x-rays via, line-emission, recombination emission and bremsstrahlung radiation [1]. The conversion efficiency of laser energy into x-ray energy depends on numerous factors such as target material or laser parameters, and can range anywhere from a fraction of a per cent to tens of per cent of the total input laser energy $[2,3,4]$. Depending on the experimental setup and spectral characteristics of the generated $\mathrm{x}$-ray radiation, relatively large amounts of this x-ray energy can be deposited within a neighbouring sample. This generally occurs on the time scale of the drive laser pulse. Most notably this technique is used within indirect drive ICF research, where gold "Hohlraums" are used to heat fusionable material to extremely high temperatures 


\section{Palladium M-L band X-rays for isochoric radiative heating of thin foil samples}

and densities [5]. In this text, however, we describe its potential use as a method for creating warm dense matter (WDM) states for further scientific exploration. Typically taken as having a solid density (1-10g/cc) and a moderate temperature $(1-100 \mathrm{eV})$, the properties of WDM are of great interest to many, especially those concerned with the structure of matter in giant planets, where WDM conditions are thought to be common [6]. There are many aspects of WDM that can be investigated experimentally, such as the equation of state [7], the electrical and thermal conductivity, and the microscopic structure [8]. Unfortunately, finding a reliable method of heating a target sample homogenously to a WDM state, ready for probing, is not trivial. WDM is also a transient state and experiments need to probe it on a short (usually $<<1 \mathrm{ns)}$ ) timescale. For example, for aluminium ionized to $Z^{*}=3$ where $\mathrm{Z}^{*}$ denotes the average charge state, the sound speed $\mathrm{Cs}$ is $\approx 3260 \sqrt{T_{e}} \mathrm{~m} / \mathrm{s}$. At $\mathrm{T}_{\mathrm{e}}=1 \mathrm{eV}$, if we assume the expansion moves at $\mathrm{Cs}$, a $1 \mu \mathrm{m}$ foil will be fully disintegrated after $\approx 300 \mathrm{ps}$. Rapid deposition of energy is crucial and radiative $\mathrm{x}$-ray heating can fulfil this criterion. Other methods to generate WDM can involve irradiation with energetic ions [9], or compression via laser driven shocks [10, 11]. For radiative heating careful consideration of the characteristics of the $\mathrm{x}$-ray source is required. The photon energy range used for heating has to be chosen to balance the efficiency and uniformity of energy deposition throughout the sample. Lower energy photons may be absorbed more efficiently, but will lead to preferential heating of the surface and a non-uniform sample. In this study we concentrate our efforts on a viable source to radiatively heat thin foils of aluminium by investigating the emission of M-L band x-rays within the $3-3.5 \mathrm{keV}$ range from palladium-coated targets. This emission is of relatively short pulse duration (sub-nanosecond) and can deposit energy before the sample expands (isochoric heating). We compare our results to previously published data from Phillion and Hailey [2]. We also consider the emission of lower energy radiation outside this spectral band, and its effects on sample heating. Target design can help suppress unwanted photon bands, and improve the uniformity of deposition.

\section{Experiment setup}

The experiment was conducted at VULCAN Target Area West at the Rutherford Appleton Laboratory, UK [12]. The experiment target setup is illustrated in figure 1. VULCAN provides up to six long pulse high energy beams at $1053 \mathrm{~nm}$. Three of these beams were frequency doubled ( $2 \omega)$ to $526.5 \mathrm{~nm}$ using KDP (type 2) crystals and focused onto one side of the target foil. The beams were incident at $6^{\circ}$ in the horizontal plane and $25^{\circ}$ degrees in the vertical plane, from target normal. Phase plates (HPP) [13] were used to give a $220 \mu \mathrm{m} \times 200 \mu \mathrm{m}$ flat top elliptical focal spot. Between the three beams, up to $110 \mathrm{~J}( \pm 5 \mathrm{~J})$ in total was provided in 200ps Gaussian FWHM pulses. This gave an intensity on the order of $10^{15} \mathrm{~W} / \mathrm{cm}^{2}$ on target. Neon-like palladium has an ionisation potential of $\approx 5.28 \mathrm{keV}$ and with electron temperatures achievable at $\approx 10^{15} \mathrm{~W} / \mathrm{cm}^{2}$ we expect copious $\mathrm{x}$-ray emission in lines from neon-like and adjacent ion stages. 


\section{Palladium M-L band x-rays for isochoric radiative heating of thin foil samples}

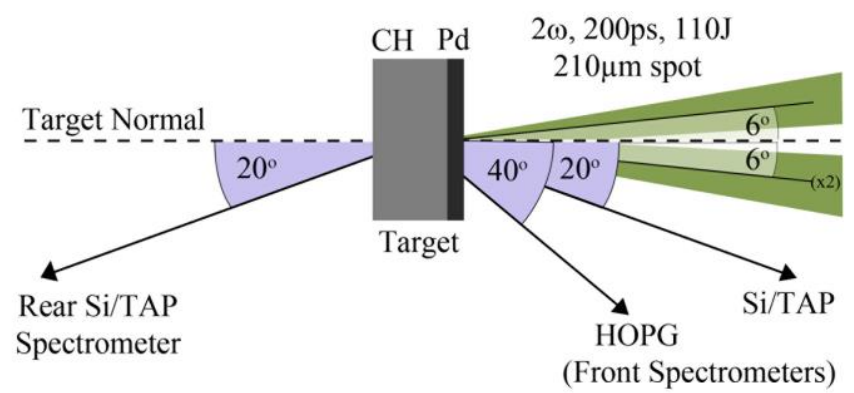

Figure 1. Experiment target setup in the horizontal plane. Laser pulses are incident from the right. Two beams are incident $25^{\circ}$ below the target normal in the vertical plane, and one beam is incident $25^{\circ}$ above the target normal.

The targets used consisted of a thin layer of palladium coated onto a $13 \mu \mathrm{m} \mathrm{CH}$ plastic substrate of parylene$\mathrm{N}$. The $\mathrm{CH}$ substrate was on the non-irradiated side, and was designed to suppress the lower energy photons generated in the interaction. The thickness of the palladium was varied between $100 \mathrm{~nm}$ and $400 \mathrm{~nm}$ to observe any change in front and rear emission.

The main diagnostics fielded to measure x-ray flux generated from the target were crystal based spectrometers. Two flat silicon (111) crystals and a flat HOPG (highly ordered pyrolytic graphite) crystal were used to monitor the palladium M-L band emission from $3.125 \mathrm{keV}$ to $3.475 \mathrm{keV}$ and two flat TAP (001) crystals were used to observe radiation between $1.465 \mathrm{keV}$ and $1.550 \mathrm{keV}$. It is important to have an accurate grasp of the emission in this region as it is close to the K-edge where aluminium is highly absorbent, i.e. it will play a large role in aluminium sample heating and may lead to non-uniform heating. On each shot two spectrometers observed the direct front emission, one at $20^{\circ}$ to the target normal ( $\mathrm{Si}$ or TAP) and another at $40^{\circ}$ to the target normal (HOPG). A third spectrometer ( $\mathrm{Si}$ or TAP) observed the rear emission at $20^{\circ}$ to the target normal. Andor DX-420BN CCDs were used as detectors and aluminium and beryllium filters were used to shield the detectors from unwanted background radiation. In line with the processing method of Phillion and Hailey, the emission is assumed to be uniformly emitted into $4 \pi$ for calculating conversion efficiency. In reality, the opacity variation of the plasma plume and target as a function of angle should be considered, but for keV x-rays these should be small corrections in the immediate vicinity of the target normal $[14,15]$. Processes which can lead to anisotropic emission of hard x-rays require laser intensities several orders of magnitude higher than those used here [16]. Equation 1 describes the method of converting CCD counts to photon source numbers.

$$
I_{s}\left(E_{p h}\right)=\frac{I_{c}\left(E_{p h}\right) g_{A D} g_{e h} 4 \pi}{T_{f}\left(E_{p h}\right) Q_{e}\left(E_{p h}\right) E_{p h} \theta_{h} \gamma_{r}\left(E_{p h}\right)}
$$

Here $I_{s}\left(E_{p h}\right)$ is the number of photons estimated to be emitted into a full sphere and $I_{\mathrm{c}}\left(E_{p h}\right)$ is the number of counts the CCDs register within a given spectral range. For the CCDs, the quantum efficiency, analogue to digital conversion (A/D gain), and the energy required to create an electron hole pair are given by $Q_{\mathrm{e}}\left(E_{p h}\right), g_{\mathrm{AD}}$, and $g_{\mathrm{eh}}$, 


\section{Palladium M-L band x-rays for isochoric radiative heating of thin foil samples}

respectively. $Q_{\mathrm{e}}\left(E_{p h}\right)$ is available from the Andor technical guide $(=85-95 \%), g_{\mathrm{eh}}=3.65 \mathrm{eV}[17]$ and $g_{\mathrm{AD}}=7.4 \pm 0.1$ electrons per count (calibrated previously using an Fe-55 radioactive source). To avoid the effects of thermal noise, the CCDs were cooled to $5^{\circ} \mathrm{C}$ on each shot. $E_{\mathrm{ph}}$ is the incident photon energy in $\mathrm{eV}$ and $T_{\mathrm{f}}\left(E_{p h}\right)$ is the total filter transmission. Variation of these factors with photon energy is accounted for in our calculations. The solid angle and thus the fraction of a full sphere that the detector subtends is given by a combination of the angle the detector sweeps out in the horizontal plane $\theta_{\mathrm{h}}$ and the rocking curve of the crystal. The term $\gamma_{\mathrm{r}}\left(E_{p h}\right)$ is the integrated reflectivity and represents the integral of the rocking curve of the crystal in use. It is important to have an accurate value for $\gamma_{\mathrm{r}}\left(E_{p h}\right)$ for each of the crystals used. We consult Henke et al [18] for our Si crystals and use the ideally perfect crystal integrated reflectivities. For the TAP crystals, from Savin et al [19], we use $\gamma_{\mathrm{r}}\left(E_{\mathrm{ph}}\right)=$ $(0.51)\left(\gamma_{\mathrm{rp}}+\gamma_{\mathrm{rm}}\right)$, where $\gamma_{\mathrm{rp}}$ and $\gamma_{\mathrm{rm}}$ are, respectively, the calculated total integrated reflectivities for photons of energy $E_{p h}$ from Henke et al for an ideally perfect crystal with absorption and for an ideally mosaic crystal. The HOPG crystal used was ZYA grade (denoting high crystalline perfection) with a mosaicity of $0.4^{\circ}$. XOP ( $x$-ray Oriented Programs) [20], an x-ray source modelling tool for optical elements, was used to calculate $\gamma_{\mathrm{r}}\left(E_{p h}\right)$ for HOPG (between 1.3mrads and $1.5 \mathrm{mrads}$ for our spectral range).

\section{Results}

\subsection{M-L band Emission}

We first consider the emission detected using the front and rear flat $\mathrm{Si}$ crystal spectrometers. Each spectrometer had $30.4 \pm 0.2 \mu \mathrm{m}$ of aluminium filtering before the crystal and a single layer of $25 \mu \mathrm{m}$ beryllium directly before the CCD. Figure 2 (a) shows an example of a raw image from the rear Si spectrometer, along with a processed lineout (b) giving the estimated photons/sphere/eV as a function of photon energy.
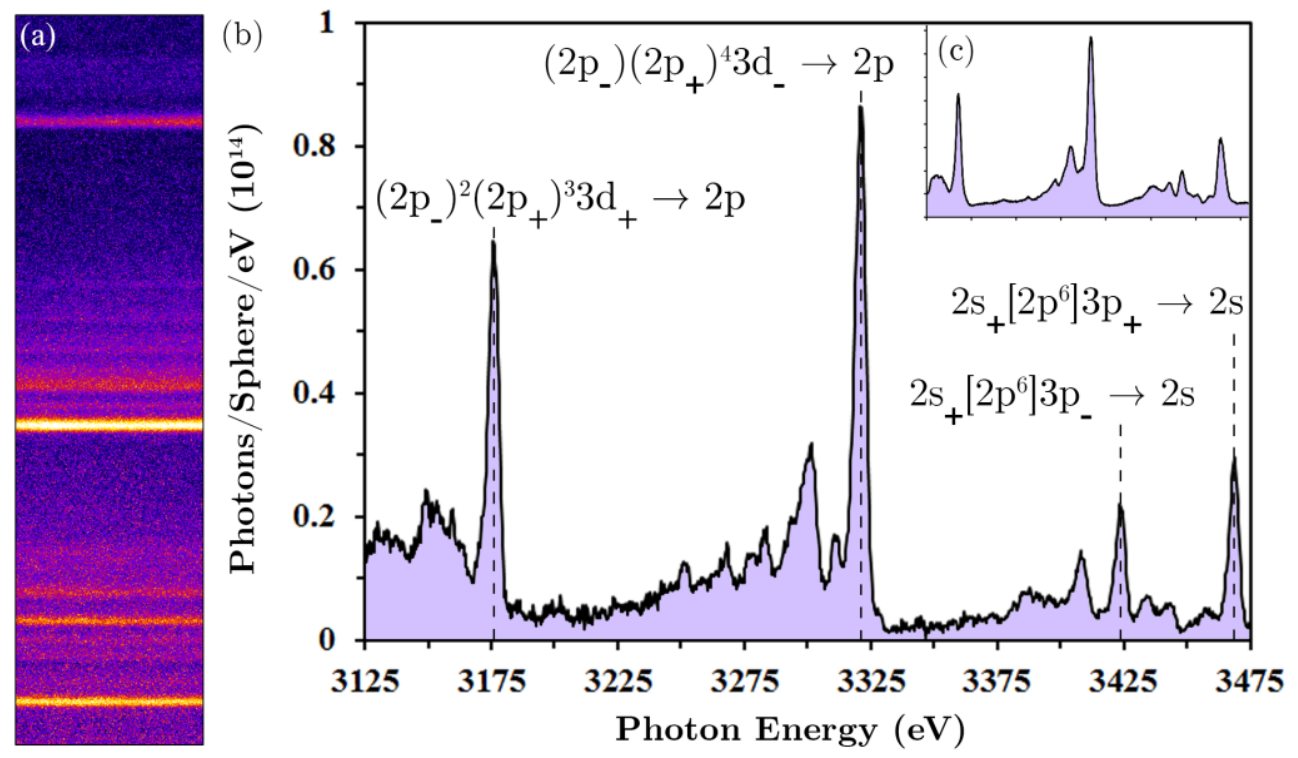


\section{Palladium M-L band X-rays for isochoric radiative heating of thin foil samples}

Figure 2. Sample rear Si crystal spectrometer data. The target was $150 \mathrm{~nm}$ Pd on $13 \mu \mathrm{m} \mathrm{CH}$. Shot $\# 2$ 7/5/2014. (a) Raw data from CCD; (b) processed lineout. The line transitions used for spectral fitting are also indicated, see text for details. These transitions, marked by dotted vertical lines, with increasing wavelength are often referred to as the 3A, 3B, 3C and 3D lines in Ne-like ion spectra designations (e.g. Hutcheon et al [21]). (c) Similar lineout profile for the lower resolution HOPG spectrometer.

Previously Phillion and Hailey identified the spectral lines shown in figure 2 as a combination of M-L band transitions in the neon-like, sodium-like, magnesium-like and aluminium-like ion stages of the plasma. They fitted line energies using available experimental data for other elements and a Moseley law scaling for palladium. For our data we have used a fit based on the work of Rice et al [22] who provides both theoretical and experimentally measured energies for these lines. The transitions identified were four of the neon-like ( $\mathrm{Pd} 36+)$ lines into the $2 s$ and $2 p$ inner-shells. These are indicated on figure 2, where for each, the terms before the arrow are the $j j$-coupled orbitals providing the upper-level designations. For the $2 p$ transitions the first two orbitals indicate the occupancy of the $2 p_{1 / 2}$ and $2 p_{3 / 2}$ subshells, respectively, and the third orbital is where the $2 p$ electron has been promoted. For the $2 \mathrm{~s}$ transitions the first orbital indicates the $2 s$ subshell vacancy, the second indicates a full (spectator) $2 p$ subshell, and third is where the $2 s$ electron has been promoted. The term after the arrow for each denotes the final inner-shell state. For all transitions shown, $J=1$ and a "-" indicates $l-s$ coupling and a "+" indicates $l+s$ coupling.

Fourteen data shots were taken in total and the key features of the results are summarized below. Figure 3 depicts the total front and total rear emission (summation of all photons/sphere between $3.125-3.475 \mathrm{keV}$ ) as a function of palladium coating thickness. The emission values are given per incident joule of laser energy, and per sphere of emission.

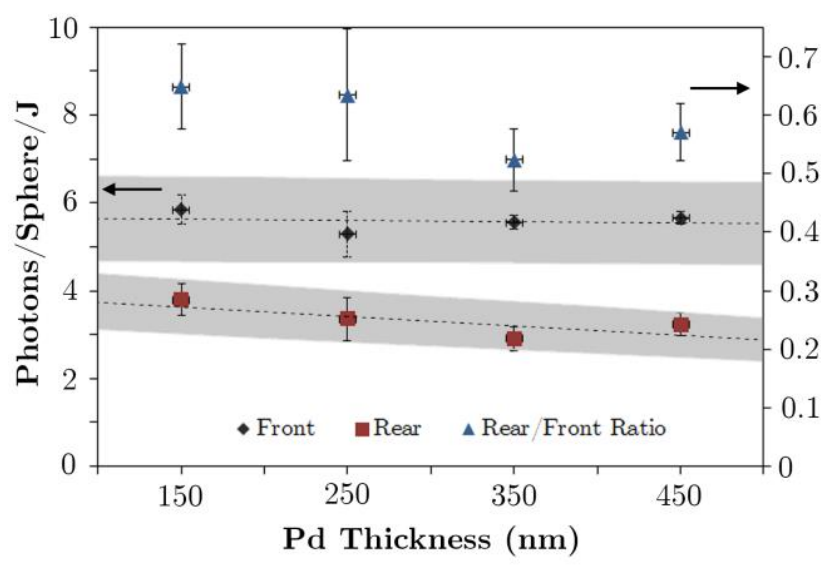

Figure 3. Mean total photons $/$ sphere $/ \mathrm{J}$ (between $3.125 \mathrm{keV}$ and $3.475 \mathrm{keV}$ ) as a function of palladium coating thickness. The front (black diamonds) and rear (red squares) are shown (left-hand vertical axis), and the ratio of these two values (rear-tofront) is also given (blue triangles, right-hand vertical axis). 


\section{Palladium M-L band x-rays for isochoric radiative heating of thin foil samples}

For each thickness, at least three shots were taken and the mean is given, with the error bars representing the standard error. The dashed straight lines are trends fitted to the mean points, and the grey regions indicate the systematic experimental error bar from this trend line. The front emission (black diamonds) can be seen to be independent of palladium thickness, whereas the rear emission (red squares) seems to be weakly dependent on the palladium thickness. The major difference here is that the rear x-rays have to penetrate any remaining target palladium and the $13 \mu \mathrm{m} \mathrm{CH}$ backing. The ratio of the rear emission to the front emission is also shown in the figure (blue triangles). The total integrated rear emission in the detected region varies from approximately $65 \%$ to $55 \%$ of the emission from the front, as the palladium thickness increases. This is very close to the combined transmission of the $\mathrm{CH}$ backing $(\approx 90 \%)$ and target palladium $(\approx 60 \%-80 \%$ for coatings of $150 \mathrm{~nm}$ to $450 \mathrm{~nm}$ respectively). This suggests the majority of the M-L band emission is occurring on the front, laser irradiated side of the palladium, where the plasma temperature and density are high, and that a substantial amount of the coating plays no part, except to attenuate the emission. Hydrodynamic simulations for the $150 \mathrm{~nm}$ coating support this, indicating that after 100ps less than one tenth of the palladium material is ionized enough and at a temperature to produce the M-L band radiation. This also explains why an increased coating thickness gives no change in the yield obtained from the front side of the target.

In figure 4 we compare the total front emission (summation of all photons/sphere between $3.125-3.475 \mathrm{keV}$ ) as a function of incident laser energy on target, for each shot. This time the error bars represent the systematic experimental error. It should be noted that the yields obtained from the secondary front viewing spectrometer (HOPG crystal) agree with those of the front $\mathrm{Si}$ spectrometer to within 7\%. This is well within the estimated experimental error bars, and gives added confidence to the measurement.

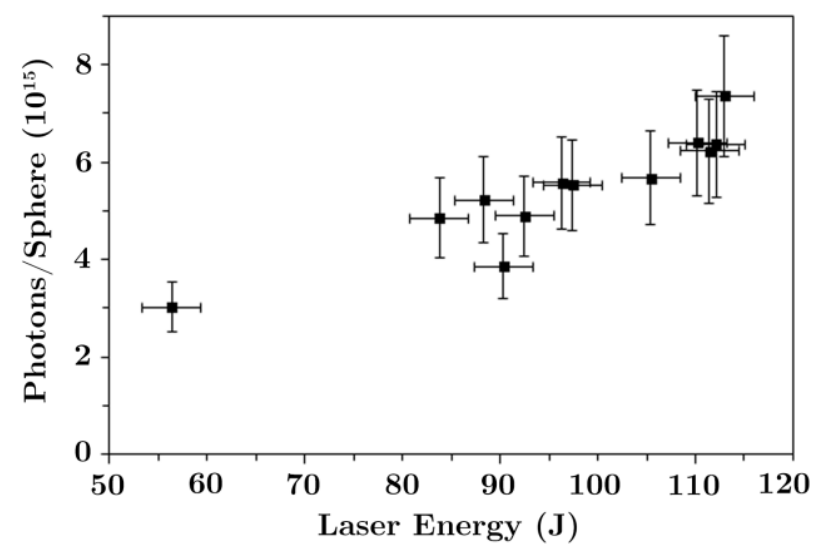

Figure 4. Total front photons/sphere (between $3.125 \mathrm{keV}$ and $3.475 \mathrm{keV}$ ) detected by the $\mathrm{Si}$ spectrometer on each shot, as a function of on-target laser energy (joules).

There is a clear increase in the total flux emitted as a function of laser energy on target. In fact, when the total flux per joule is considered, an increase in conversion efficiency is still seen. Figure 5 depicts the number of total Photons/Sphere/J (conversion efficiency) recorded by Phillion and Hailey, alongside values measured within our 


\section{Palladium M-L band x-rays for isochoric radiative heating of thin foil samples}

experimental setup, again as function of incident laser energy. The spectral window of our data $(3.125 \mathrm{keV}-$ $3.475 \mathrm{keV})$ is narrower than that of Phillion and Hailey $(2.9 \mathrm{keV}-3.55 \mathrm{keV})$. Integrating the given spectra from Phillion and Hailey as a function of photon energy indicates approximately $75 \%$ of their total photon signal is contained with our smaller spectral limits. Thus for direct comparison our values were scaled accordingly (i.e. multiplied by a factor of 1.33 ).

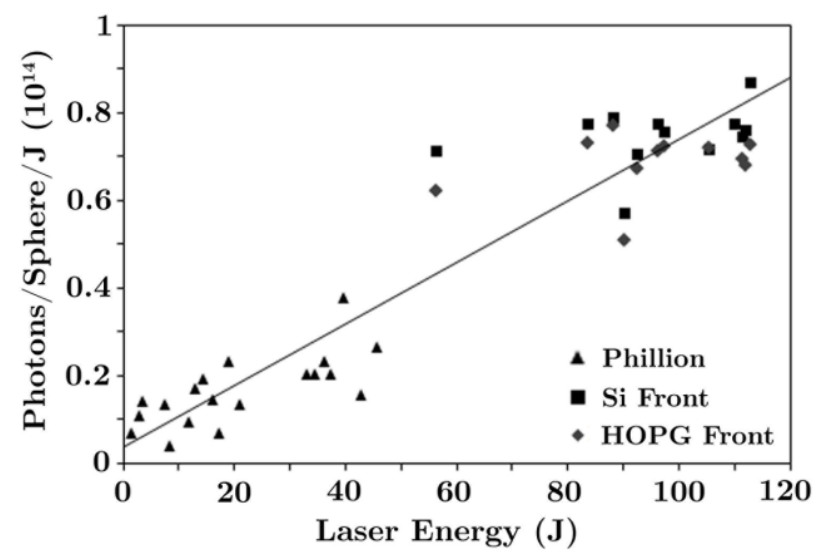

Figure 5. Conversion efficiencies (Photons/Sphere/J) as a function of laser energy (joules) from Phillion and Hailey (triangles) where the spectral window is $2.9 \mathrm{keV}-3.55 \mathrm{keV}$, and our front Si spectrometer (squares) and HOPG spectrometer (diamonds) scaled to the same spectral window ( $33 \%$ increase from the original window of $3.125 \mathrm{keV}-3.475 \mathrm{keV}$ ).

The maximum conversion efficiency seen in our data, nearly $9 \times 10^{13}$ photons/sphere/joule, is over twice the most efficient emission detailed in Phillion and Hailey $\left(3.78 \times 10^{13}\right.$ photons/sphere/joule). This increase in conversion efficiency follows the trend indicated by Phillion and Hailey's data (highlighted by the straight line fit in figure 5). The connection between pulse duration and conversion efficiency to keV line emission in this regime has been discussed in detail [3] and was found to be connected to the generally increased absorption at longer pulse durations that comes about through the production of a longer scale-length plasma where inverse bremsstrahlung is more efficient. This was seen to offset the higher fraction of the laser energy that is converted to kinetic energy of the expanding plasma rather than thermal energy for longer pulses.

\subsection{Lower energy Emission}

We now consider the emission detected between $1.465 \mathrm{keV}$ and $1.550 \mathrm{keV}$ with the front and rear TAP spectrometers. Only one useable data shot is present here. The second spectrometer on the front (HOPG), verifies that the x-ray yield in the M-L band region is very similar to that of the shots with the Si spectrometers present, and it is assumed emission is comparable to these shots. Figure 6 shows an example of a raw image from the front TAP spectrometer (a), along with a processed lineout (b) giving CCD counts as a function of photon energy. 
Palladium M-L band x-rays for isochoric radiative heating of thin foil samples
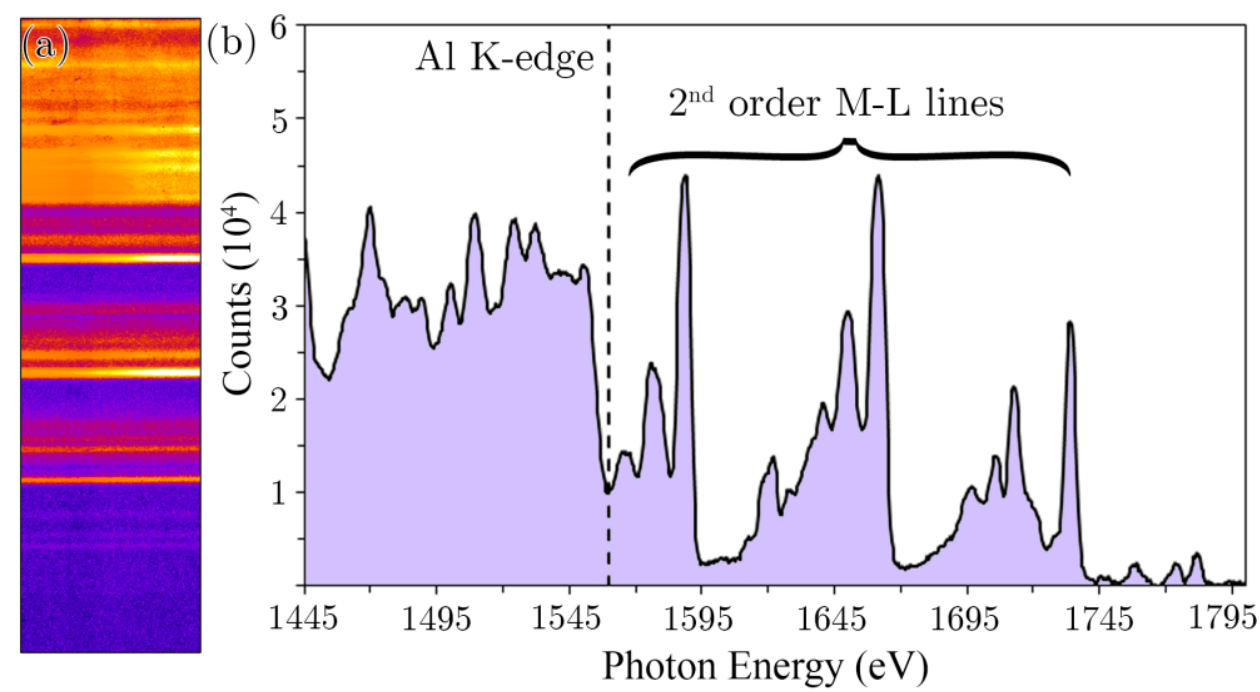

Figure 6. Front TAP spectrometer data. The target was 150nm Pd on $13 \mu \mathrm{m} \mathrm{CH}$. Shot \#2 9/5/2014 (a) Raw data from CCD (b) lineout.

This time the spectral dispersion is fitted using the second order M-L band lines, which can be seen clearly in the raw counts lineout. The aluminium $\mathrm{K}$-edge at $1.5596 \mathrm{keV}$ is also highlighted in figure 6 with the vertical dashed line. Due to the levels of aluminium filtering on the spectrometer, no counts on the higher energy side of this edge should be from $1^{\text {st }}$ order photons $\left(\approx 10^{-14}\right.$ transmission $)$. Before processing the data to photon yield as a function of photon energy, the influence of the second order signal was removed. An estimation of the second order counts below the K-edge was inferred using the second order signal above the K-edge. An exponential fit to the M-L lines between $3.12-3.18 \mathrm{keV}$ (seen in figure 6 between $1.56-1.59 \mathrm{keV}$ ) was extended into the region below the Kedge (depicted in figure 8). This exponential fit was seen to match the shape of the first order emission when using the Si spectrometers. Figure 7 depicts the remaining counts between $1.465 \mathrm{keV}$ and $1.550 \mathrm{keV}$, for both front and rear spectrometers, converted to photons/sphere/eV using equation 1. It should be noted that some of the peak structures left in the data are a remnant of the $2^{\text {nd }}$ order signal, but contribute a small fraction to the total signal in the region.

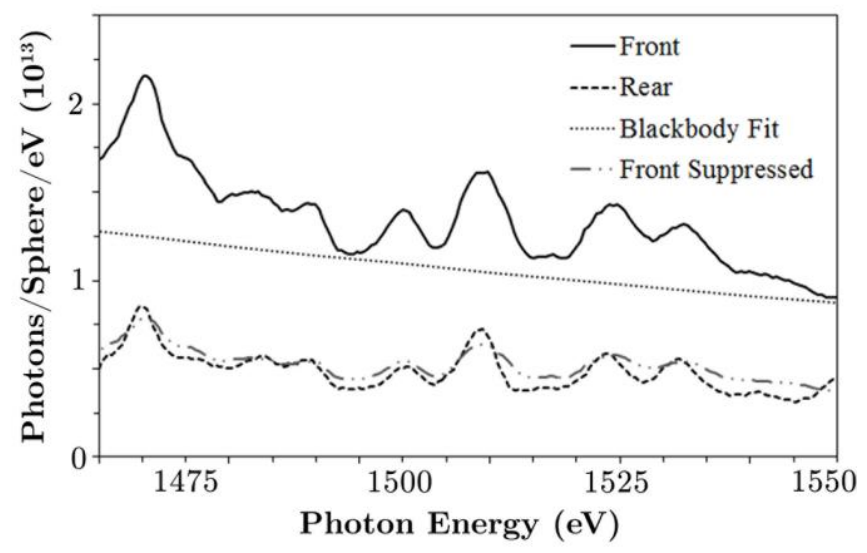




\section{Palladium M-L band X-rays for isochoric radiative heating of thin foil samples}

Figure 7. Front (solid) and rear (dashed) photons/sphere/eV between 1465 and $1550 \mathrm{eV}$. Also depicted is the blackbody fit detailed in the text (dotted), and the front data suppressed by $13 \mu \mathrm{m} \mathrm{CH}$ (dash-dotted).

There is quite high broadband emission within the detector region. In fact it is comparable in yield to the emission in the higher M-L band. However, the detected rear emission (dashed line on figure 7) is clearly much lower than that from the front. This is due to the $\mathrm{CH}$ plastic backing suppressing the emission. In fact the total rear yield in comparison to the total front yield seems to directly reflect the transmission of $\mathrm{CH}$ in this region (36-42\%). The broadband emission is due to bremsstrahlung from within the target combined with lower energy line transitions. Hydrodynamic simulations show the necessary conditions for this emission exist throughout a large extent of the palladium, unlike the M-L band emission which is confined to the hotter region close to the laser interaction. Hence, the palladium $(150 \mathrm{~nm}$ coating here) seems to play no major role in differentially transmitting the broadband emission from the front or rear. Overlaid on figure 7 is the front data if it was suppressed by $13 \mu \mathrm{m}$ of $\mathrm{CH}$. The scaled emission matches the rear data extremely well.

In an attempt to model the radiation outside of our detector spectral regions, a blackbody distribution has been fitted to the front TAP data. If we assume the radiation is emitted from an area equal to the laser spot size, and follows a similar pulse duration to that of the laser pulse, the spectral radiance is matched for a blackbody temperature of $\approx 175 \mathrm{eV}$. Figure 7 depicts the blackbody distribution fit to the front TAP data. This is quite a high blackbody temperature, but not unreasonable considering the intensity of our target interaction. The StefanBoltzmann law can be used to calculate the total power radiated by the surface, which is directly proportional to the fourth power of the temperature. For our on target laser intensity (nearly $2 \times 10^{15} \mathrm{~W} / \mathrm{cm}^{2}$ ), a distribution based on a temperature of $175 \mathrm{eV}$ infers approximately $5 \%$ conversion from laser light into overall broadband emission. This is a very reasonable estimate.

Figure 8 depicts the full spectra estimated to be emitted from the rear side of the foils. The blackbody distribution has been suppressed by the transmission of $13 \mu \mathrm{m} \mathrm{CH}$ (dashed), with a plot of the matched region inset, and the real data from the rear TAP and Si spectrometers can also be seen (solid). Note the scaling difference for the M-L band region (right-hand vertical axis), to aid visualization. 
Palladium M-L band x-rays for isochoric radiative heating of thin foil samples

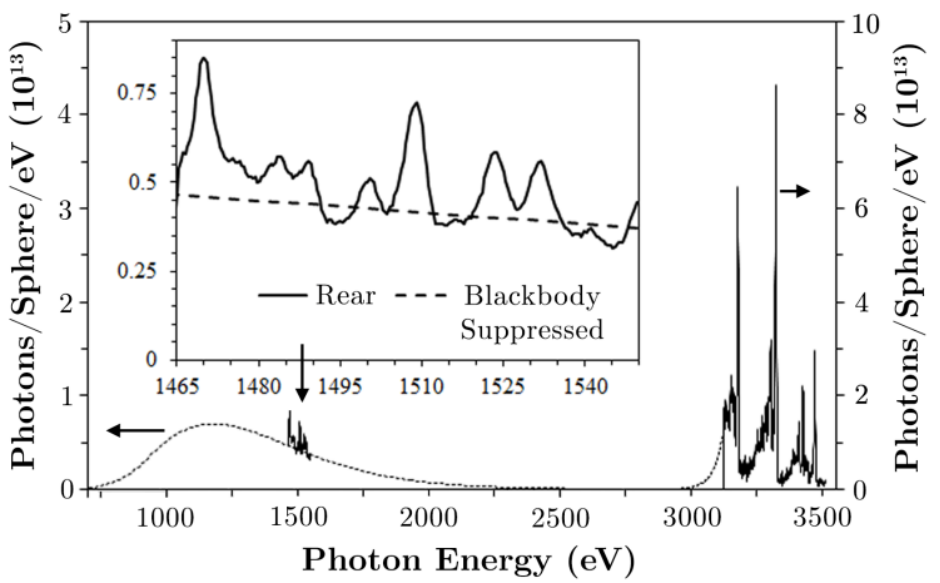

Figure 8. Full emission spectra from the rear side of the foils. Solid lines indicate data from the rear TAP and Si spectrometers. Dashed lines indicate the modelled emission from a suppressed blackbody distribution and the exponential fitting to the M-L band emission. A closer comparison of the rear TAP data to the suppressed blackbody distribution can be seen inset.

Future experiments will field spectrometers to observe a wider window of lower energy emission $(0.5-1 \mathrm{keV}$ region). This should help build a more accurate model of the spectra and total radiation flux.

\subsection{Temporal emission}

During a second experiment with a virtually identical setup to that described, a Kentech x-ray streak camera [23, 24] was used to measure the pulse duration of the M-L band emission. See figure 9. A 200nm CsI cathode coated on a $25 \mu \mathrm{m}$ beryllium substrate was used, and the diagnostic had additional filters of $8 \mu \mathrm{m}$ silver and $55 \mu \mathrm{m}$ Kapton (polyimide). The combined transmissions of these filters (inset in figure 9) block any optical light and soft x-rays, allowing only the palladium M-L band radiation to pass. The pulse duration of the x-rays is found to be very similar to that of the incident laser, as expected. An asymmetric Gaussian was fitted with a FWHM of $\approx 195 \pm 10$ ps. The rise time of the x-ray pulse is seen to be steeper than the fall off. This more gradual fall off is also expected as the plasma plume expands and cools. 
Palladium M-L band $x$-rays for isochoric radiative heating of thin foil samples

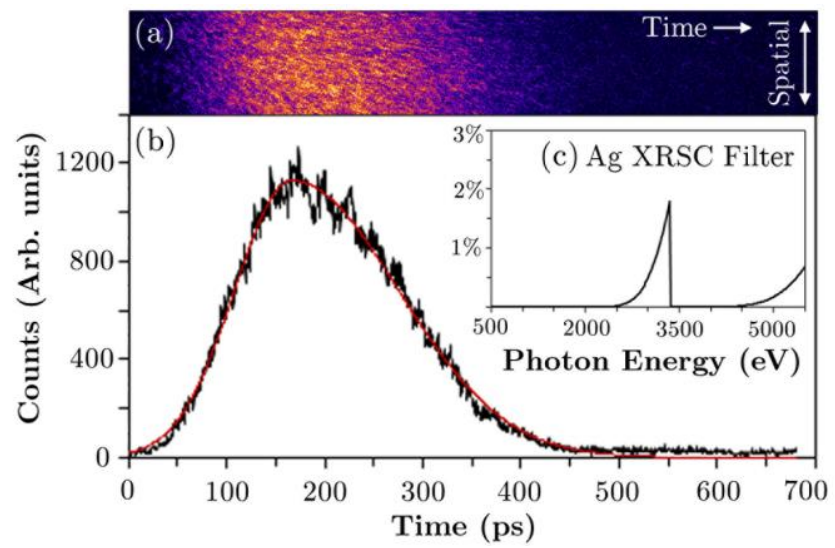

Figure 9. Pulse duration measurements using an x-ray streak camera (XRSC). (a) Raw data. (b) Averaged lineout. The red line indicates an asymmetric Gaussian fitted to the data. FWHM $\approx 195 \pm 10$ ps. (c) Inset; transmission profile of XRSC filters.

\subsection{X-ray source size}

Also present on the second experiment was an x-ray pinhole camera. Four $25 \mu \mathrm{m}$ pinholes in a tantalum substrate were placed $85 \mathrm{~mm}$ from the source, and an Andor DX-435 CCD was placed a further $353 \mathrm{~mm}$ from the pinholes. This setup relayed four images of the source spot to the CCD with a magnification of $\approx 4.2$. Separate filters were placed over the pinholes to create various spectral channels. The transmission of one of these channels $(8.75 \mu \mathrm{m}$ cadmium) can be seen in figure 10 (c); the filter selects only the Pd M-L band x-rays. Harder x-rays ( $>5 \mathrm{keV})$ will also penetrate but these are expected to be much weaker than the L-shell line radiation. Another of these filters used aluminium to allow radiation around the $\mathrm{Al} \mathrm{K}$-edge to be observed. For this channel an estimate of the M-L band x-rays contained in the spot (to which the aluminium is not opaque) is subtracted from the signal. This estimation is provided by the cadmium channel. The x-ray source sizes were compared to the focal spot size of the incident laser. Due to the relatively large size of the laser spots they were recorded by placing a CCD camera ( $9 \mu \mathrm{m}$ pixels) directly at the focus of the beam. For these shots only two of the three beams were available to use. See Figure 10 (a) and (b).
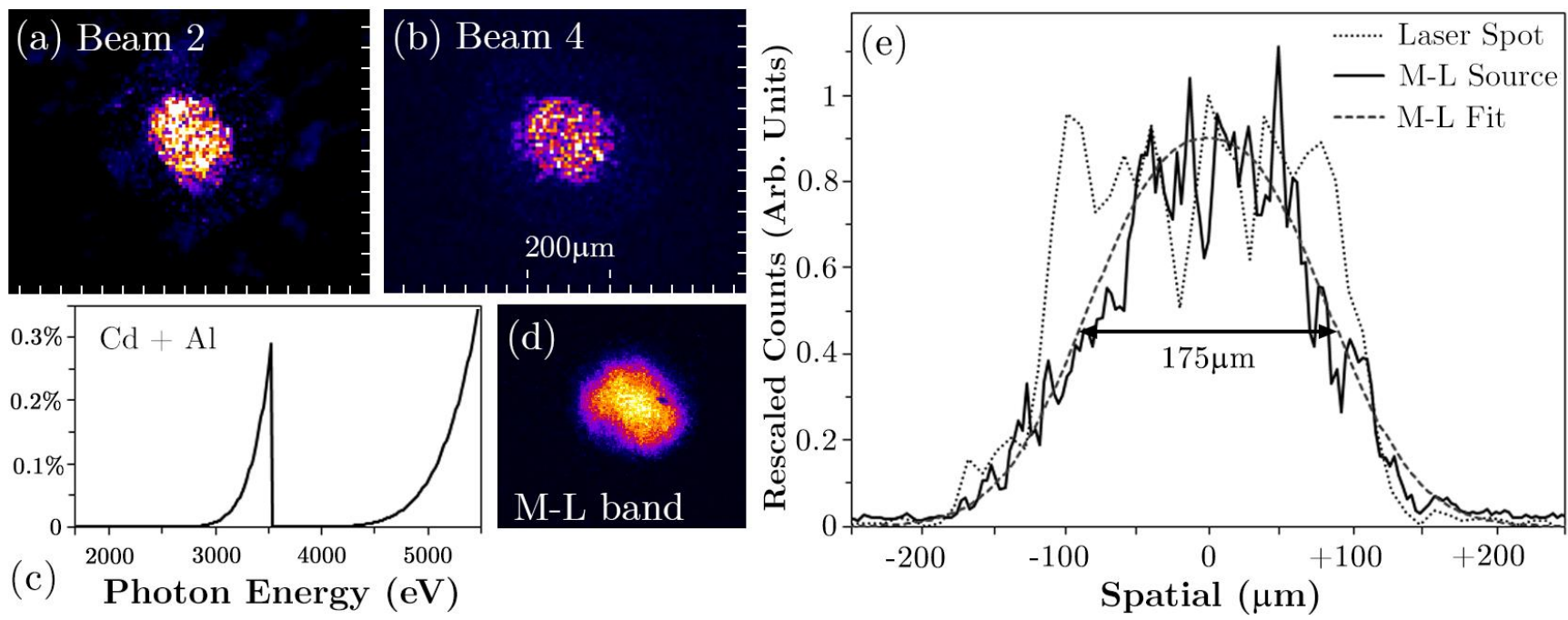
Palladium M-L band x-rays for isochoric radiative heating of thin foil samples

Figure 10. (a) \& (b) Laser focal spots of beams 2 and 4, with the phase plates in place (c) Transmission profile for the cadmium filter channel (d) Pinhole image of the M-L band x-ray emission (e) Spatial lineout comparisons of the laser focal spot to the M-L band x-ray emission. A super-Gaussian fit can be seen for the M-L band.

In general the shape and size of the x-ray emission is found to follow the laser focal spot quite closely. However, the intensity profile of the x-ray emission does differ. See figure 10 (e) for a comparison of the laser focal spot to the M-L band x-ray source. Super-Gaussian curves were fitted to both the more top-hat shaped laser focal spots and the more peaked x-ray emission. The non-flat-top x-ray profile is most likely connected with the 2D expansion at the edge of the plasma plume and lateral thermal transport. For our laser intensities, hydrodynamic simulations predict the electron temperature at the critical density to be $4-5 \mathrm{keV}$, and we can estimate that at the peak of the laser pulse, the density scale length is of the order of $\approx 50 \mu \mathrm{m}$. This means that there would be some deviation from 1-D behaviour with enhanced expansion and cooling at the edges, leading to a drop in x-ray yield.

Due to the elliptical shape of our laser focal spots (a combination of the incident laser angle and the phase plate shape) the source size measurements were performed along the major and minor axes. Due to an obscuration of the signal on some shots, a $70^{\circ}$ angle measurement (see figure 10 (a)) was also made where the major and minor line outs were not possible. A comparison of the FWHMs of both the Al K-edge and the Pd M-L band x-ray sources with the laser focal spot can be seen in figure 11. All three axes are assessed and data from a low power $(20 \mathrm{~J})$ and full power $(60 \mathrm{~J})$ shot is given.
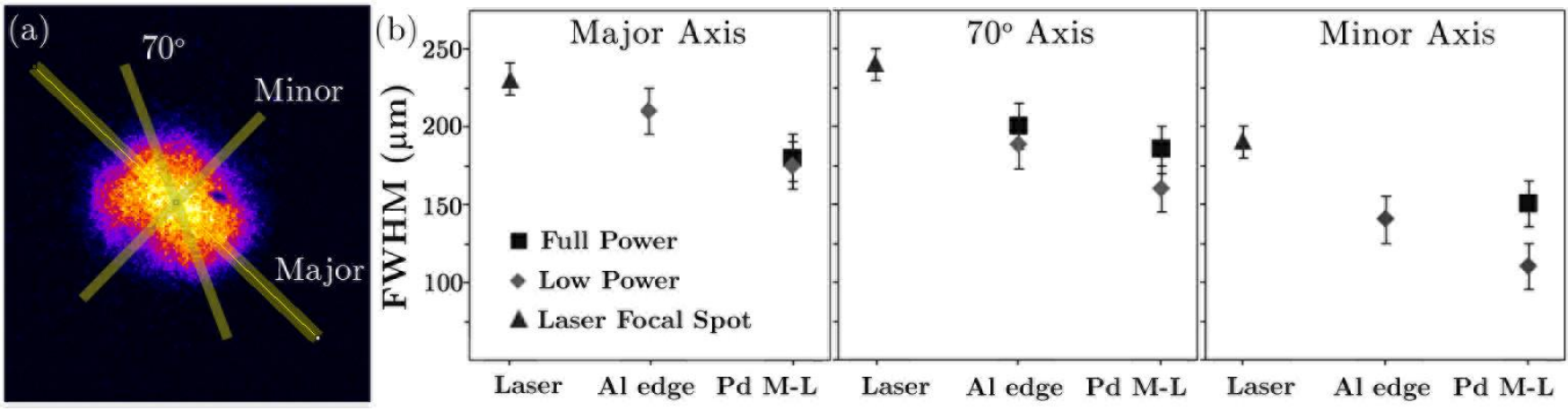

Figure 11. (a) Depiction of the lineout axes (b) Comparison of the measured FWHMs of the laser focal spot, the Al K-edge, and the Pd M-L band sources, for each axis (major, minor and $70^{\circ}$ ).

It is clear that the X-ray sources are smaller than the laser focal spots. It is also evident that the lower energy Al Kedge x-rays are generated in a bigger source than the higher energy Pd M-L band x-rays. This is again most likely due to the expansion of the plasma plume and cooling at the edge, as a higher temperature is required for the M-L band generation.

\section{Isochorically heating thin foil aluminium}




\section{Palladium M-L band X-rays for isochoric radiative heating of thin foil samples}

We propose the use of the palladium heater targets described above for the heating of a thin foil aluminium sample to WDM conditions for probing. As mentioned in the introduction, the x-ray photon energy range of the M-L band palladium emission between $3-3.5 \mathrm{keV}$ is chosen in an attempt to balance the efficiency and uniformity of the absorption, and hence deposition of energy throughout the sample. Higher energy photons may penetrate the target more easily, leading to a more uniform absorption across the depth of the sample. However, the corresponding absorption would of course be lower, and it becomes increasingly difficult to provide a high enough flux to heat the sample to the desired level.

A possible experimental layout for a future experiment can be seen in figure 12. A $0.8 \mu \mathrm{m}$ thick aluminium foil sample is placed between two $\mathrm{CH}$ backed palladium foil heater targets, each $1 \mathrm{~mm}$ from the sample. The rear emission from the palladium heater targets is taken to follow typical results outlined previously in section 3 . The sample is tilted at $45^{\circ}$, allowing a line of sight for probing and a presented area of flux for absorbing the heater xrays. Diagnostics to monitor the conditions produced may include; Streaked optical pyrometry (SOP) to infer sample temperature [25, 26], surface velocity measurements (VISAR) to deduce target expansion [27], or even xray near edge spectroscopy (XANES) to investigate target temperature and structure [28]. The experimental layout proposed here is designed to provide a possible technique to investigate the free-free opacity of aluminium in the WDM state for the XUV regime by measuring the absorption of a separate high harmonic XUV probe, also pictured in figure 12 .

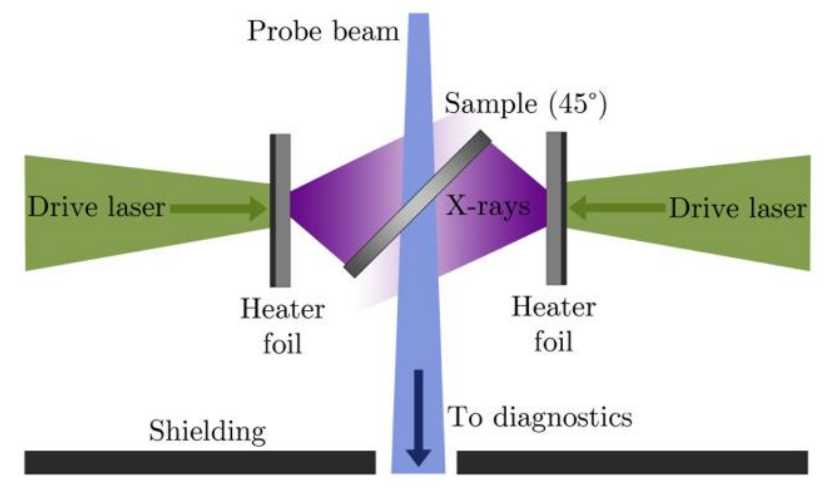

Figure 12. Suggested experimental layout; a palladium heater foil $1 \mathrm{~mm}$ either side of a sample target, tilted at $45^{\circ}$. A straight line of sight for probing is available.

The emission from the heater foils must be accurately modelled to ensure sufficient deposition, and to determine whether the deposition will be uniform enough for probing. Ray tracing simulations suggest that given the emission model described, for a $200 \mu \mathrm{m}$ x-ray spot size either side, the central $250 \mu \mathrm{ms}$ of the sample foil will have $\approx 12 \%$ variation in energy deposition laterally across the target. As a function of depth through the target the uniformity can vary from $1 \%$ variation at the centre to $8 \%$ at the edges. 


\section{Palladium M-L band x-rays for isochoric radiative heating of thin foil samples}

The energy deposition described is temporally integrated across the duration of the x-ray source. In order to ensure a WDM state, this deposition must take place in a short enough time scale and before the sample begins to expand and the density drops. Using the spectral and temporal emission profiles given in section 3, HYADES [29] (a 1-D radiation hydrodynamics code) simulations were carried out to estimate the sample temperature and density as a function of time. Various photon source groups were defined to model the incoming flux on a $0.8 \mu \mathrm{m}$ aluminium sample, irradiated from both sides, as depicted in figure 12. Although only true within the limitations mentioned above, as HYADES is a 1-D code, the energy deposition was taken as spatially uniform. The results of this simulation are shown in figure 13. A more detailed discussion of the modelling will be forthcoming in a separate paper.
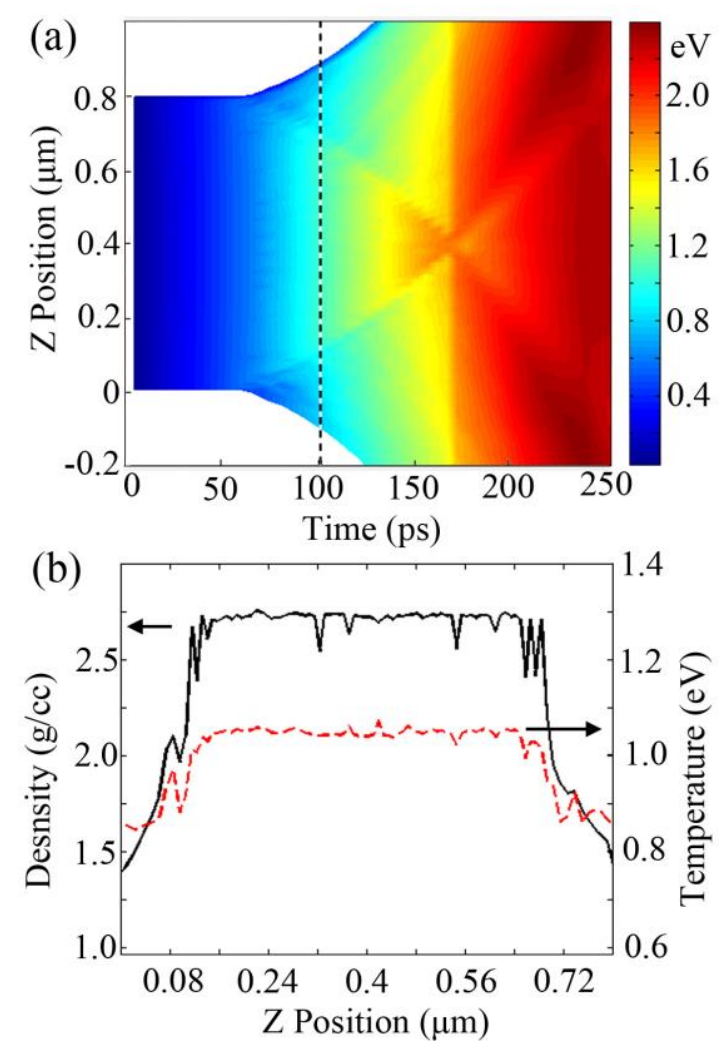

Figure 13. (a) HYADES results for the emission model outlined. The vertical axis is spatial and represents the foil thickness. The horizontal axis is temporal, zero being the onset of the photon flux source. The electron temperature (eV) is given by the colour bar. (b) A snapshot at 100ps giving the density (solid line) and electron temperature (dotted red line) though the foil.

The target temperature increases rapidly from the onset of the x-ray heating pulse, and the foil is seen to steadily expand. Selecting an appropriate probe time allows various conditions to be measured. Figure 13(b) depicts a snapshot $100 \mathrm{ps}$ after the onset of the laser pulse. Approximately $80 \%$ of the foil remains at solid density and has been heated to over $1 \mathrm{eV}$. Probing before this time would provide a more solid foil at lower temperature, whereas 


\section{Palladium M-L band x-rays for isochoric radiative heating of thin foil samples}

probing later would provide a more uniform yet less dense and cooler condition. After approximately 200ps, the density of the foil is seen to halve $(1.35 \mathrm{~g} / \mathrm{cc})$, but heated to over $2.2 \mathrm{eV}$. If this level of heating is sufficient, the use of a thicker $\mathrm{CH}$ backing on the palladium targets would help suppress lower energy photons and should help improve sample target uniformity.

\section{Conclusion}

We have characterized the front and rear M-L band x-ray emission from palladium coated plastic heater targets, irradiated with a high energy laser pulse, and witnessed higher conversion efficiencies than previously reported. We have also measured radiation around the aluminium K-edge, and modelled emission surrounding this region. By using the plastic substrate as a means of suppressing lower energy photons, we have investigated the possibility of using the rear emission of these palladium targets as a radiative x-ray heater source. Finally, although not yet experimentally tested, simulations show promising results for using this technique to rapidly heat a thin aluminium foil sample before expansion takes place, thus creating a WDM state for probing.

\section{Acknowledgements}

This work was supported by the Engineering and Physical Sciences Research Council (grant numbers EP/I031464 and EP/I029206/1), and the Science and Technology Facilities Council (STFC), UK. We acknowledge the support and contribution of the Target Preparation Laboratory, Vulcan laser staff and the Engineering workshop at CLF, RAL.

\section{References}

[1] Gibbon P 2005 Short Pulse Laser Interactions with Matter (Imperial College Press) ISBN: 978-1-86094-135-1

[2] Phillion D W and Hailey C J 1986 Phys. Rev. A 34 4886-96

[3] Riley D, Woolsey N C, McSherry D, Khattak F Y, Weaver I 2002 Plasma Sources Sci. Technol. 11 484-491

[4] Matthews D Let al 1983 Journal of Appl. Phys. 54, 4260-4268

[5] Lindl J 1995 Phys. Plasmas 2 3933-4024

[6] Alfe D, Gillan M J, Price G D 2007 Contemporary Physics 48, 63-80

[7] Renaudin P, Blancard C, Clérouin J, Faussurier G, Noiret P and Recoules V 2003 Phys Rev. Lett. 917 075002-1

[8] White S et al 2013 High Energy Density Physics 9 573-577 


\section{Palladium M-L band X-rays for isochoric radiative heating of thin foil samples}

[9] Patel P K et al 2003 Phys. Rev. Lett. 91125004

[10] Trainor R J, Shaner J W, Auerbach J M and Holmes N C 1979 Phys. Rev. Lett. 42 1154-1157

[11] Yoo C S, Holmes N C, Ross M, Webb D J and Pike C 1993 Phys. Rev. Lett. 70 3931-3934

[12] http://www.clf.stfc.ac.uk/CLF/Facilities/Vulcan/12248.aspx

[13] Kato Y, Mima K, Miyanga N, Arinaga S, Kitagawa Y, Nakatsuka M, Yamanaka C 1984 Phys. Review Lett. 53 $1057-1060$

[14] Kania D R et al 1992 Phys. Rev. A 46 7853-68

[15] Shang W, Zhang W, Dong Y, Huang C, Zhu T, Song T, Yang J 2013 Journal of Alloys and Compounds 578, 1-4

[16] McKeever K et al 2015 Phys. Rev. E 91033107

[17] Owens A, Fraser G W, Abbey A F, Holland A, McCarthy K, Keay A and Wells A 1996 Nuc. Inst. and Meth. in Phys. Res. A 382, 503-510

[18] Henke B L, Gullikson E M, and Davis J C 1993 Atomic Data and Nuclear Data Tables 542 181-342

[19] Savin D W et al 1996 The Astrophysical Journal 470 L73-L76

[20] Sánchez Del Río M, Ferrero C, Mocella V 1997 Proc. SPIE 3152 148-157

[21] Hutcheon R J, Cooke L, Key M H, Lewis C L S, Bromage G E 1980 Physica Scripta 21 89-97

[22] Rice J E, Fournier K B, Terry J L, Graf M A, Finkenthal M, Marmar E S, and Goldstein W H 1996 Phys. Rev. A 53 $63953-62$

[23] Bradley D J, Roddie A G, Sibbett W, Key M H, Lamb M J, Lewis C L S, Sachsenmaier P 1975 Optics Communications $152231-236$

[24] Murnane M M, Kapteyn H C, Falcone R W 1990 Appl. Phys. Lett. 561948

[25] Miller J E, Boehly T R, Melchior A, Meyerhofer D D, Celliers P M, Eggert J H, Hicks D G, Sorce C M, Oertel J A and Emmel P M 2007 Rev. Sci. Inst. 78034903

[26] Ni P A, Bieniosek F M, Henestroza E and Lidia S M 2014 Nuc. Inst. and Meth. in Phys. Res. A 733 12-17

[27] Celliers P M, Bradley D K, Collins G W, Hicks D G, Boehly T R and Armstrong W J 2004 Rev. Sci. Inst. 7511

4916-29

[28] Lévy A et al 2009 Plasma Phys. Control. Fusion 51124021

[29] Larsen J T and Lane S M 1994 J. Quant. Spec. Radiat. Transfer 51 179-186 